\title{
Cutaneous leishmaniasis in a returning traveller
}

\author{
Eric Demers PhD, David M. Forrest MD MHSc, Gabriele E. Weichert MD
}

A n otherwise healthy 42-year-old man presented with a small erythematous nodule on the superior part of his right ear and substantial swelling of the entire pinna (Figure 1A), which he had first noticed 2 weeks before presentation. There were no other lesions, palpable lymphadenopathy or systemic symptoms. Eleven weeks before his initial presentation, the patient, a biologist, had conducted a university field school in Belize, Central America, which included travel in rainforest habitats. None of the other 23 members of the group had similar cutaneous lesions, although 5 had acquired botfly infestations.

The condition was initially treated as a bacterial infection with two 7-day courses of antibiotics (cloxacillin and cephalexin), with no response. Over a period of 10 weeks, the nodule and surrounding area became progressively ulcerated, with purulent exudates and crusting of most of the pinna superior to the ear canal (Figure 1B). Giemsa stain of a skin biopsy showed several intracellular and free, small, oval bodies (amastigotes) consistent with Leishmania species (Figure 2). In vitro culture and polymerase chain reaction analysis identified Leishmania mexicana.

Initial antileishmanial treatment with liposomal amphotericin B was administered daily by intravenous infusion over 5 days $(5 \mathrm{mg} / \mathrm{kg}$ on day $1,3 \mathrm{mg} / \mathrm{kg}$ on days $2-5$; total dose 17 $\mathrm{mg} / \mathrm{kg}$ ). Little improvement ensued over a 3week (Figure 1C) and 6-week (Figure 1D) period, which suggested that the treatment regimen had been ineffective. Consequently, oral fluconazole $(400 \mathrm{mg} / \mathrm{d})$ was started and continued for 56 days. Because there was no apparent change in the lesion during the first 25 days of this treatment, twice-weekly intralesional injections of the pentavalent antimonial compound sodium stibogluconate were given at a dosage of $50 \mathrm{mg} / \mathrm{cm}^{2}$ ( $2 \mathrm{~mL}$ per injection) for 5 weeks. Except for some considerable localized pain during injection, no major adverse effects were observed. There was marked improvement of the lesion after 3 weeks (Figure 1E) and 5 weeks (Figure 1F) of this treatment.

\section{Discussion}

Cutaneous leishmaniasis is endemic to many tropical and subtropical regions of the world, with worldwide incidence of about 1.5 million new cases each year. ${ }^{1}$ The condition is caused by protozoan parasites of the Leishmania genus, which are transmitted from infected mammals to humans through the bite of phlebotomine sandflies. When promastigote stages are injected by blood-feeding sandflies into the mammalian host, they invade macrophages and transform into amastigotes, which replicate extensively within a parasitophorous vacuole. ${ }^{2}$ In the case of cutaneous forms of leishmaniasis, the amastigotes multiply in the reticuloendothelial system of macrophages of the skin around the initial bite wound, eventually producing a relatively large sore or ulcer. Although the cutaneous disease is not as severe as mucocutaneous and visceral forms of leishmaniasis, it can have substantial consequences, including permanent scarring, disfigurement and possible parasite dissemination.

This cutaneous presentation of leishmaniasis endemic in the rainforests of Central America is commonly referred to as the "chiclero ulcer" or in Belize as the "bay sore." Cutaneous leishmaniasis caused by L. mexicana typically heals spontaneously within a few months. Complications arise when lesions are associated with the cartilaginous pinna of the ear because of its poor vascularization and limited immune response. Leishmanial lesions of the ear may last for several months to many years and may cause severe mutilation of the pinna if left untreated.

\section{KEY POINTS}

- Early diagnosis of cutaneous leishmaniasis is important to ensure early treatment and to limit mutilation or scarring.

- Referral to an infectious disease specialist should be considered for selection of appropriate treatment.

- Local therapy may be an appropriate mode of treatment.

- With climate change, leishmaniasis may be seen more frequently in North America.
Competing interests: None declared.

This article has been peer reviewed

Correspondence to:

Eric Demers,

Eric.Demers@viu.ca

CMAJ 2013. DOI:10.1503 /cmaj.120694 


\section{Diagnosis}

Traditionally, the diagnosis of leishmaniasis has relied heavily on a history of travel to a diseaseendemic region, typical clinical features, microscopic identification of amastigotes by histology or direct microscopy of biopsy smears or aspirates, and the growth of promastigotes in culture. ${ }^{1}$ The
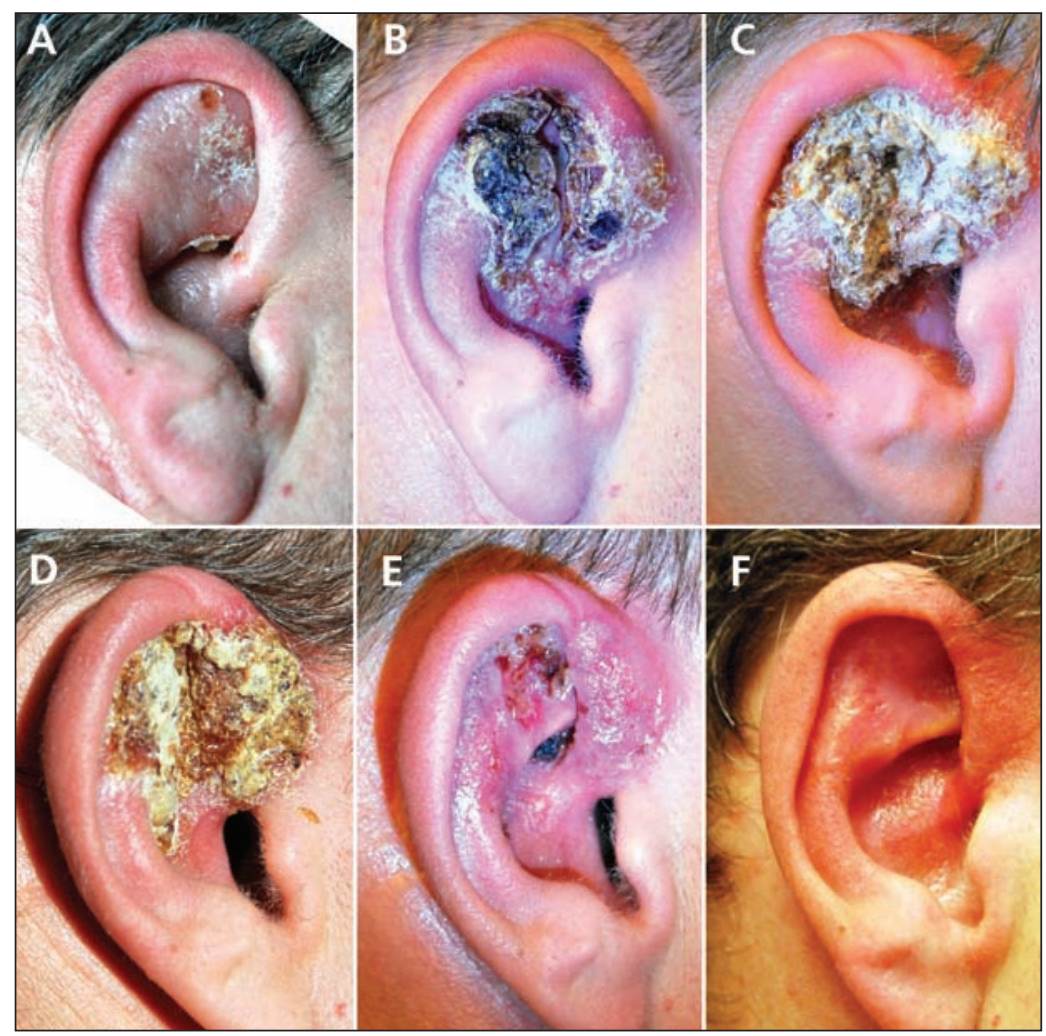

Figure 1: External manifestation of cutaneous leishmaniasis in a 42-year-old man. Photographs depict (A) initial presentation and thereafter at intervals of (B) 10 weeks, (C) 13 weeks, (D) 16 weeks, (E) 22 weeks and (F) 24 weeks.

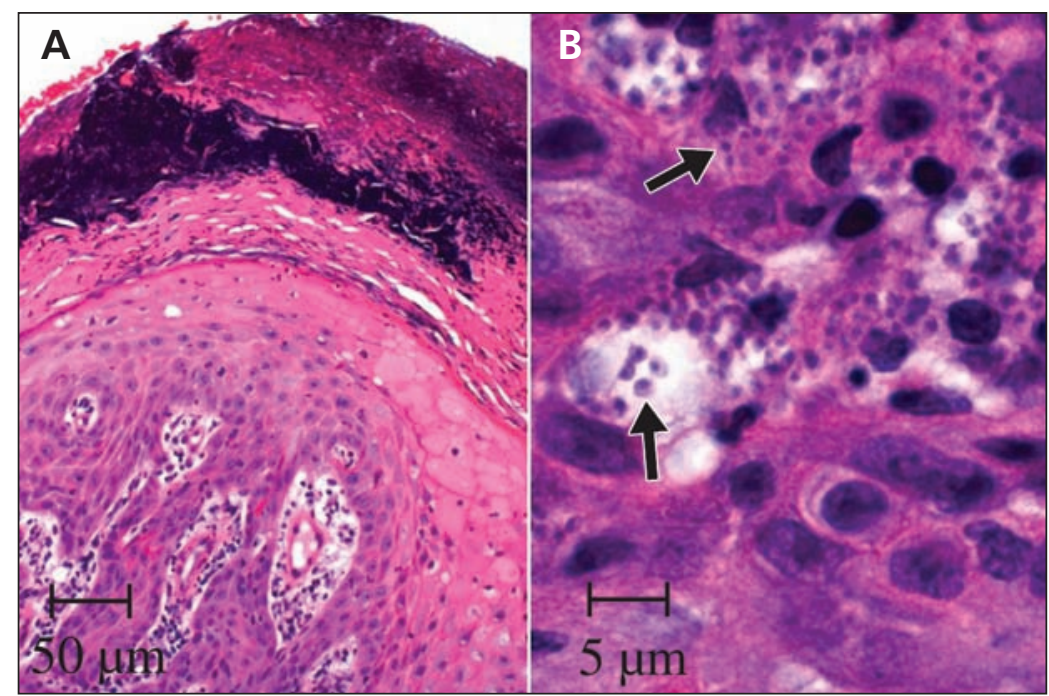

Figure 2: Geimsa stain of a biopsy specimen of the ear ulcer. (A) Epidermal and dermal tissue showing impetiginized hyperkeratosis (original magnification $\times 100$ ). (B) Multiple intracellular amastigotes (arrows) of Leishmania mexicana in parasitophorous vacuoles of histiocytes (original magnification $\times 1000$ ). sensitivity of these techniques, however, can be variable, depending on parasite number and dispersion in samples, technical expertise and media used to culture the parasite. Polymerase chain reaction testing has a specificity of up to $100 \%$, and $20 \%$ $30 \%$ greater sensitivity than conventional methods. ${ }^{1}$

Because of the broad clinical spectrum of cutaneous leishmaniasis, its long incubation period and resemblance to common skin diseases such as bacterial or fungal infections and nonmelanoma skin cancers, diagnosis is often delayed. Early diagnosis is important, because the parasite replicates rapidly and the lesion can grow substantially once established. In addition, because some Leishmania species (e.g., L. braziliensis and L. panamensis) have a propensity for mucosal spread, ${ }^{1}$ delayed diagnosis may lead to the need for complicated and potentially toxic treatments. Furthermore, because treatment for various species of Leishmania may be different, delayed or improper identification of the condition may lead to suboptimal treatment, prolonging the course of the disease and resulting in disfiguring scars. ${ }^{4}$

\section{Treatment}

The therapeutic arsenal against leishmaniasis is relatively limited, and the absence of consistent clinical practice guidelines in North America can constrain its effective treatment.

Pentavalent antimonial compounds have been the therapeutic drugs of choice for the treatment of localized cutaneous leishmaniasis for more than 50 years. ${ }^{5}$ The World Health Organization (WHO) recommends treating cutaneous leishmaniasis with pentavalent antimonial drugs at $20 \mathrm{mg} / \mathrm{kg}$ daily intramuscularly or intravenously for 20-28 consecutive days. ${ }^{6}$ A meta-analysis of 23 studies reported a cure rate of $76.5 \%$ among a total of 1133 patients. $^{7}$ These drugs have not been approved for general sale in Canada and must be obtained through Health Canada's Special Access Programme.

The polyene antibiotic amphotericin B represents an alternative treatment for cutaneous leishmaniasis, especially formulations with reduced toxicity such as liposomal amphotericin B. ${ }^{5}$ In 1997, the US Food and Drug Administration approved liposomal amphotericin B for the treatment of visceral leishmaniasis at a dose of $3 \mathrm{mg} / \mathrm{kg}$ daily for 7 doses. ${ }^{8}$ Despite the common use of liposomal amphotericin B in the treatment of the visceral form of leishmaniasis, ${ }^{1}$ there have been no controlled studies investigating its use in the treatment of cutaneous leishmaniasis. A small retrospective study reported a cure rate of $84 \%$ (16 of 19 patients) with the initial treatment regimen; the remaining patients were cured with additional dosing. ${ }^{8}$ 
Although oral treatment with azole compounds (e.g., fluconazole) has shown variable success rates $(30 \%-89 \%$ cure rates) for Leishmania species, their ease of administration and well-studied safety profile make them frequently used agents for cutaneous leishmaniasis. ${ }^{1}$

Intralesional injections with sodium stibogluconate have been shown to be effective for the treatment of cutaneous leishmaniasis and are better tolerated than systemic therapy with antimonial compounds. ${ }^{9}$ For local therapy of $L$. mexicana, WHO recommends $1-5 \mathrm{~mL}$ of antimonial compounds (1-5 infiltrations) administered every 3-7 days for 3-4 weeks. ${ }^{6}$ In a cohort study, 220 patients with $L$. tropica lesions in India received intralesional injections of sodium stibogluconate in doses of $50 \mathrm{mg} / \mathrm{cm}^{2}$ weekly or twice weekly for 3-4 weeks. ${ }^{9}$ Cure rates of $92 \%$ and $96 \%$, respectively, were observed, and there were no relapses in cured patients reported up to 2 years after treatment. The most common adverse effect was localized pain and edema within 24 hours of injection. ${ }^{9}$

\section{Cutaneous leishmaniasis in returning travellers}

Increased travel to regions where cutaneous leishmaniasis is endemic has been associated with an increase in cases of the disease in returning travellers, with many of these cases related to travel to Latin America. ${ }^{4}$ Although there has been no systematic review of imported cutaneous leishmaniasis to Canada, some reports have been published..$^{10,11}$ Because Canadians have increased their travel to Central and South America by an average of $14 \%$ annually between 2003 and $2010,,^{12}$ the occurrence of cutaneous leishmaniasis in returning Canadian travellers may also increase.

In Central and South America, transmission of leishmaniasis is traditionally restricted to rainforest habitats. ${ }^{13}$ Travellers to these habitats (e.g., ecotourists, biologists) may be unaware that they have acquired the parasite. The variable incubation period of the disease (typically 2 weeks to 6 months $)^{1}$ may delay clinical manifestations until after travellers have returned to their home country, which can pose a diagnostic challenge for clinicians who may be unfamiliar with the disease's manifestations. Therefore, emergence of cutaneous leishmaniasis in the developed world warrants increased awareness from primary care physicians. In addition, lack of ready availability and access to appropriate antiparasitic therapy may present an obstacle in treatment. Referral to an infectious disease specialist should be considered for the selection of appropriate treatment.

Travellers can reduce the risk of infection by protecting themselves from sandfly bites, especially from dusk to dawn, when sandflies gener- ally are the most active. Such protection includes wearing clothing that minimizes the amount of exposed skin, using bed netting and applying insect repellent to exposed skin. Sandflies are susceptible to insecticide repellents that contain the chemical DEET (diethyltoluamide). ${ }^{\prime}$

In the future, presentation of this disease may also increase in nontravellers. Climate change is expected to exacerbate the ecological risk of human exposure to leishmaniasis in the southern United States and, possibly, parts of southern Canada. ${ }^{13}$ This points to the importance of more widespread knowledge of this condition among health care providers, increased surveillance for leishmaniasis in the US and Canada, and ready availability of antimonial compounds for treatment.

\section{References}

1. Reithinger R, Dujardin JC, Louzir H, et al. Cutaneous leishmaniasis. Lancet Infect Dis 2007;7:581-96.

2. Alexander J, Satoskar AR, Russell DG. Leishmania species: models of intracellular parasitism. J Cell Sci 1999;112:2993-3002.

3. Vargas-Gonzalez A, Canto-Lara SB, Damian-Centeno AG, et al. Response of cutaneous leishmaniasis (chiclero's ulcer) to treatment with meglumine antimoniate in southeast Mexico. Am J Trop Med Hyg 1999;61:960-3.

4. Blum JA, Hatz CF. Treatment of cutaneous leishmaniasis in travelers 2009. J Travel Med 2009;16:123-31.

5. Almeida OLS, Santos JB. Advances in the treatment of cutaneous leishmaniasis in the new world in the last ten years: a systematic literature review. An Bras Dermatol 2011;86:497-506.

6. World Health Organization. Control of the leishmaniasis: report of a meeting of the WHO Expert Committee on the Control of leishmaniases; 2010 Mar. 22-26; Geneva. Geneva (Switzerland): The Organization; 2010.

7. Tuon FF, Amato VS, Graf ME, et al. Treatment of New World cutaneous leishmaniasis - a systematic review with a metaanalysis. Int J Dermatol 2008;47:109-24.

8. Wortmann G, Zapor M, Ressner R, et al. Lipsosomal amphotericin B for treatment of cutaneous leishmaniasis. Am J Trop Med Hyg 2010;83:1028-33.

9. Bumb RA, Mehta RD, Ghiya BC, et al. Efficacy of short-duration (twice weekly) intralesional sodium stibogluconate in treatment of cutaneous leishmaniasis in India. Br J Dermatol 2010;163:854-8.

10. Haider S, Boutross-Tadross O, Radhi J, et al. Cutaneous ulcer in a man returning from Central America. CMAJ 2003;168:590-1.

11. Abadir A, Patel A, Haider S. Systemic therapy of New World cutaneous leishmaniasis: a case report and review article. Can J Infect Dis Med Microbiol 2010;21:e79-83.

12. International travel. Ottawa $(\mathrm{ON})$ : Statistics Canada; modified 2013. Available: www5.statcan.gc.ca/bsolc/olc-cel/olc-cel?catno =66-201-X\&chropg=1\&lang=eng (accessed 2012 Mar. 5)

13. González C, Wang O, Strutz SE, et al. Climate change and risk of leishmaniasis in North America: predictions from ecological niche models of vector and reservoir species. PLoS Negl Trop Dis 2010;4:e585.

Affiliations: From the Biology Department (Demers), Vancouver Island University; Nanaimo Regional General Hospital (Forrest); and Brickyard Medical Clinic (Weichert), Nanaimo, BC

Contributors: All of the authors contributed to the conception and design of the manuscript. Eric Demers drafted the article, which David Forrest and Gabriele Weichert revised critically for important intellectual content. All of the authors gave final approval of the version submitted for publication.

Acknowledgement: The authors acknowledge Tim Goater for his contribution regarding the biology of the Leishmania parasite and for his editorial assistance in preparing the manuscript for submission. 